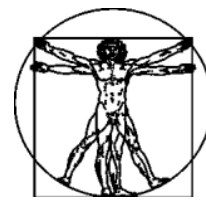

\title{
ТЕЧЕНИЕ ВОЗДУХА В НОСОВОЙ ПОЛОСТИ ЧЕЛОВЕКА. РЕЗУЛЬТАТЫ МАТЕМАТИЧЕСКОГО МОДЕЛИРОВАНИЯ
}

\author{
В.Л. Ганимедов, М.И. Мучная, А.С. Садовский \\ Институт теоретической и прикладной механики им. С.А. Христиановича Сибирского отделения \\ Российской академии наук, Россия, 630090, Новосибирск, ул. Институтская, 4/1, e-mail: mim@itam.nsc.ru
}

\begin{abstract}
Аннотация. Сложное строение носовой полости человека практически исключает изучение течения воздуха в ней с помощью экспериментальных методов визуализации и диагностики потоков, поэтому в настоящее время для этой цели активно используется численное моделирование. Эти исследования являются актуальными ввиду развития ингаляционных методов введения лекарственных препаратов, с их помощью можно проводить виртуальные операции. В институте теоретической и прикладной механики им. С.А. Христиановича Сибирского отделения Российской академии наук разработана методика исследования течения воздуха в носовой полости человека на основе математического моделирования с помощью пакета ANSYS. Трехмерная геометрия носовой полости строится на базе полученных из клиники серий томографических снимков. При построении используются программы GRAPHER и GAMBIT. Течение в построенной геометрической модели носовой полости рассчитывается в газодинамическом разделе FLUENT пакета ANSYS на основе системы осредненных по Рейнольдсу уравнений Навье-Стокса для несжимаемого газа, используется модель $k-\omega$ турбулентности. С помощью метода установления находится квазистационарное решение для заданного перепада давления между входом в носовую полость и выходом из неё. По полученным от медиков сериям томографических снимков были построены модели носовой полости для тридцати взрослых людей. Отмечены чрезвычайное разнообразие форм и, как следствие, существенные различия в структуре потока. Результаты расчетов течения воздуха анализируются с целью поиска закономерностей и индивидуальных особенностей. Проведено сравнение интегральных характеристик, таких как зависимость удельного расхода воздуха от перепада давления, распределение минимального и среднего значения температуры по длине носовой полости в зависимости от температуры вдыхаемого воздуха. Показано, что для определения фризиологической нормы недостаточно знать только расходные характеристики носовой полости, необходимо также учитывать терморегулирующую функцию.
\end{abstract}

Ключевые слова: численное моделирование, носовая полость человека, структура течения, визуализация поля течения, респираторное усилие, распределение температуры.

\section{ВВЕДЕНИЕ}

Математическое моделирование позволяет подробно исследовать структуру течения воздуха в носовой полости, что недоступно современным инструментальным

(C) Ганимедов В.Л., Мучная М.И., Садовский А.С., 2015

Ганимедов Владимир Леонидович, к.ф.-м.н., старший научный сотрудник лаборатории физических проблем управления газодинамическими течениями, Новосибирск

Мучная Мария Ивановна, к.ф.-м.н., старший научный сотрудник лаборатории физических проблем управления газодинамическими течениями, Новосибирск

Садовский Алексей Сергеевич, аспирант, лаборант лаборатории физических проблем управления газодинамическими течениями, Новосибирск 
методам. Оно дает возможность проводить виртуальные операции и прогнозировать результаты реального хирургического вмешательства. Оно может помочь в определении способа адресной доставки лекарств при ингаляции.

Исследования течения воздуха в носовой полости человека и животных с помощью численного моделирования ведутся с начала 90-х гг. ХХ в. Им посвящено большое число зарубежных публикаций, см. [3, 4, 6-11] и библиографию к ним. География публикаций весьма обширна: от США и Канады до Австралии, от Англии и Германии до Китая и Японии. Благодаря Интернету большинство работ, опубликованных, как правило, в специфических изданиях, доступны в России. Развитию данного направления способствовали совершенствование томографии и распространение коммерческих программных средств, в частности, газодинамического пакета FLUENT, который в настоящее время входит в программный комплекс ANSYS.

Первые расчеты проводились для модели одной половины носовой полости, и число объемных ячеек расчетной сетки не превышало ста тысяч [6]. В более поздних работах течение вохдуха моделируется для всей носовой полости с включением части носоглотки, и расчетные сетки насчитывают до нескольких миллионов объемных элементов. В ряде работ численные исследования выполнены для идеализированных симметричных моделей; так, авторы статьи [4] и предшествующих ей работ рассматривают на таких моделях влияние, которое оказывает на течение изменение геометрических размеров и форм различных частей носовой полости. Большинство же работ посвящено изучению течения воздуха для моделей реальных носовых полостей. Структура течения для одной модели носа конкретного человека подробно рассматривается в работе [11] для двух значений удельного расхода воздуха, соответствующих спокойному и глубокому дыханию. Также для одной модели в работе [7] рассчитываются скорость и температура потока, отмечена связь между областями высокой температуры внутри носа с образованием вихрей малой интенсивности; результаты расчетов хорошо согласуются с измерениями in vivo. В работе [9] по результатам расчетов течения воздуха в четырёх моделях носовой полости здоровых людей показаны значительные межиндивидуальные различия в структуре течения. Интернациональный коллектив авторов работы [3] исследует особенности течения воздуха в носу пациента, страдающего атрофическим ринитом; воздушные и тепловые потоки для моделей носовой полости до и после операции сравниваются с потоками в носовых полостях четырёх здоровых людей и делаются рекомендации для практики хирургического вмешательства. Результаты междисциплинарного исследования [8] показали, что виртуальная операция на основе численного моделирования может быть полезна как часть дооперационного планирования для пациентов с многофакторными нарушениями анатомии носовых ходов. В работе [10] проведены расчеты для 24 построенных моделей носовых полостей, получены поля скорости и температуры, сделана попытка найти интегральную зависимость между температурой и геометрическими параметрами.

Первая отечественная публикация на тему исследования течения воздуха в носовой полости человека на основе пакетной технологии [1] появилась в 2010 г. В настоящей работе представлен анализ результатов численных исследований, проведенных на основе обширного по клиническому спектру томографического материала. Количество рассмотренных носовых полостей позволило констатировать чрезвычайное разнообразие форм и, как следствие, существенные различия в структуре потока. Результаты расчетов анализируются в целях поиска закономерностей, в том числе для выявления признаков физиологической нормы и патологии. Установлено, что в формировании потока решающую роль играют индивидуальные анатомические особенности. Показано, что игнорирование этого факта может привести к нежелательным последствиям при хирургическом вмешательстве. 


\section{ПОСТАНОВКА ЗАДАЧИ}

В институте теоретической и прикладной механики им. С.А. Христиановича Сибирского отделения Российской академии наук разработана методика исследования течения воздуха в носовой полости человека с помощью пакета ANSYS. Трехмерная геометрия носовой полости строится на базе полученных из клиники серий снимков в коронарных сечениях, выполненных на томографе с шагом 1-5 мм. Томографический материал был получен из трёх источников: Новосибирский научный исследовательский институт травматологии и ортопедии, Новосибирская областная клиническая больница и Научный исследовательский институт патологии кровообращения им. Е.Н. Мешалкина. Все данные были переданы анонимно или с согласия пациентов.

Принята левая прямоугольная система координат, в которой ось $Z$ направлена от входа перпендикулярно коронарным сечениям, а ось $Y$ - вертикально вверх. При построении геометрии используются программы GRAPHER и GAMBIT. Течение воздуха в построенной геометрической модели носовой полости рассчитывается с помощью газодинамического решателя FLUENT пакета ANSYS 12. Решение строится на основе системы осредненных по Рейнольдсу уравнений Навье-Стокса для несжимаемого газа, дополненных полуэмпирической моделью турбулентности $k-\omega$ [12]. На поверхности носовой полости ставятся условия прилипания для скорости и постоянства температуры.

С помощью метода установления находится стационарное решение для заданного перепада давления между входом в носовую полость и выходом из неё. Константа установления для всех компонент равна $10^{-5}$. Заметим здесь, что в настоящей работе, как и в большинстве работ по данной тематике, вдох и выдох рассматриваются как квазистационарные состояния при некотором осредненном значении респираторного усилия, соответствующем вдоху или выдоху. Такой подход используется при исследовании в условиях спокойного дыхания, к режиму учащенного дыхания он неприменим.

В процессе решения находятся давление $p$, три компоненты скорости $V_{X}, V_{Y}, V_{Z}$ и температура $T$. Систему уравнений и подробности постановки задачи можно найти в [1]. Расчетная сетка в области решения содержала до $6 \times 10^{6}$ объемных ячеек. Тестовые расчеты показали, что дальнейшее сгущение сетки не вносит заметных изменений в параметры потока.

Расчеты проводились для перепадов давления от 2 до 150 Па, которые соответствуют диапазону дыхания от едва различимого (слабого) до интенсивного. При этих значениях перепада давления режим течения воздуха в носовой полости может быть как ламинарным (при малом респираторном усилии), так и развитым турбулентным (при большом респираторном усилии), а также может иметь переходный характер. Экспериментальные исследования, выполненные в [5] на модели носовой полости с двадцатикратным увеличением с использованием критериев подобия, позволили авторам сделать вывод, что при большинстве значений расхода течение воздуха является умеренно турбулентным. В работе [13] отмечено, что течение воздуха в верхних дыхательных путях человека имеет характер переходного режима: в каких-то областях течение может быть локально ламинарным, а в других - локально турбулентным. На основании численного эксперимента в этой работе показано, что для описания подобных течений в каналах лучше всего подходит адаптированная для малых чисел Рейнольдса LRN (Low Reynolds Number) модель турбулентности $k-\omega[12]$. В настоящей работе в расчетах использовалась именно эта модель.

Достоверность результатов численных расчетов принято подтверждать в сравнении с экспериментом. В отсутствие результатов прямых измерений параметров течения воздуха в носовой полости сравнение было проведено с данными, получаемыми 


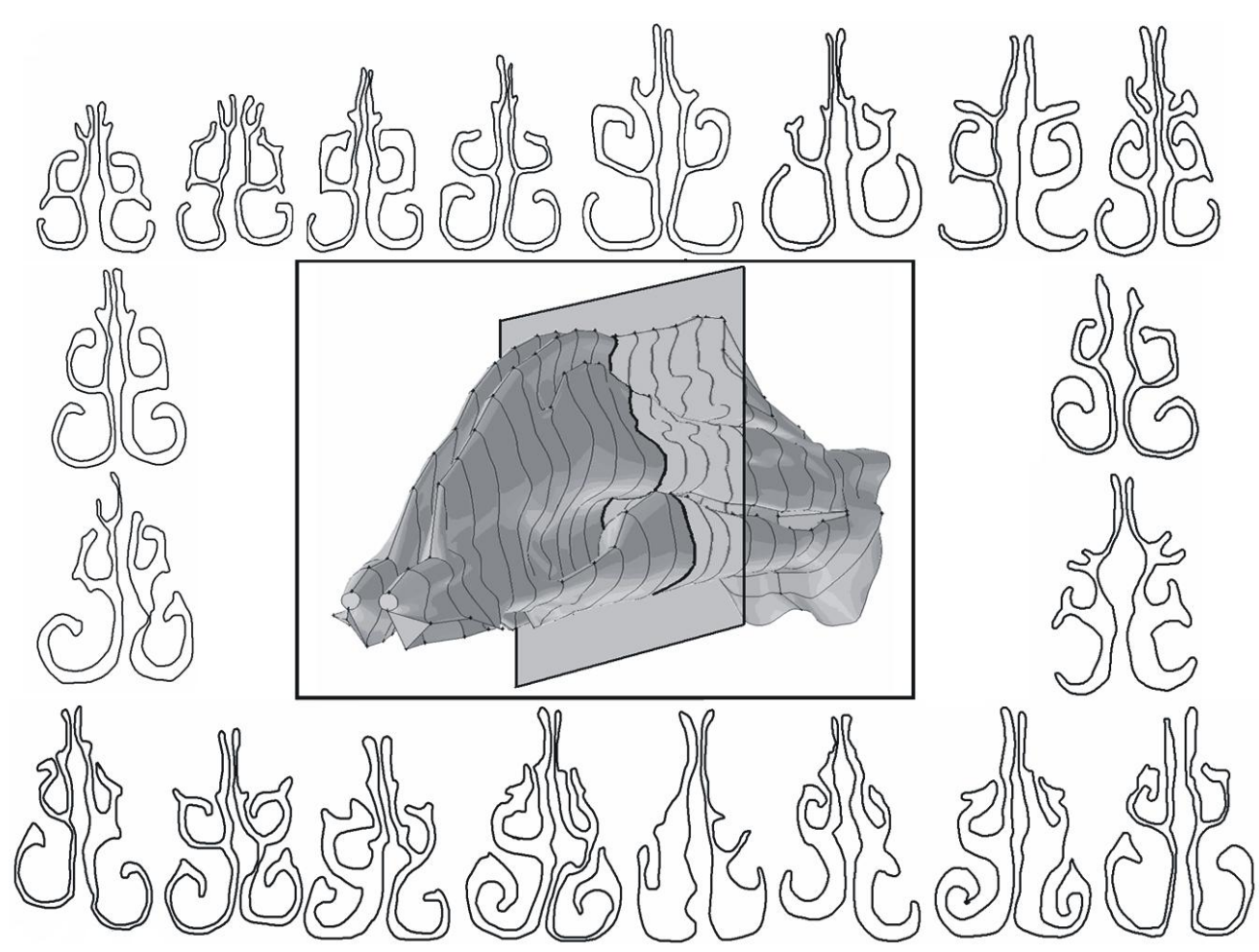

Рис. 1. Разнообразие форм носовой полости

с помощью клинического метода передней активной риноманометрии. Для этого были численно смоделированы условия проведения измерений и осуществлены расчеты течения, соответствующие этим условиям [2]. Сравнение зависимостей удельного расхода воздуха от респираторного усилия показало хорошее соответствие рассчитанных и измеренных данных, что позволяет надеяться на адекватность результатов, получаемых с помощью разработанной технологии численного моделирования.

\section{РАЗНООБРАЗИЕ ФОРМ}

На основе полученных от медиков серий томографических снимков были построены модели носовой полости для тридцати взрослых людей. Отмечено чрезвычайное разнообразие форм. На рис. 1 показаны характерные коронарные сечения двадцати носовых полостей (сечения относятся к средней части носового лабиринта). В центре рисунка представлен общий вид поверхности носовой полости одной из моделей и показан след от коронарного сечения в средней части носового лабиринта. Из рисунка видно, что все рассматриваемые модели имеют индивидуальные анатомические особенности в строении носовой полости и сильно отличаются друг от друга. Часть из них (нижний ряд) представляет носовые полости, характеризуемые медиками как патологические случаи. Правая и левая половины носовой полости конкретного человека, как правило, имеют заметные отличия, особенно значительные для моделей, относящихся к патологии. Более наглядно различия между правой и левой половинами носовой полости, а также различия носовых полостей разных людей демонстрирует рис. 2. Здесь приведена визуализация поля продольной компоненты скорости в носовой полости при вдохе для перепада давления $\Delta p=50$ Па в ряде последовательных коронарных сечений для шести человек. Можно видеть, что нижняя граница носовой полости может быть расположена почти горизонтально, а также с наклоном от ноздрей до хоаны: отрицательным или положительным, причем в двух последних случаях наклон сильно выражен. 

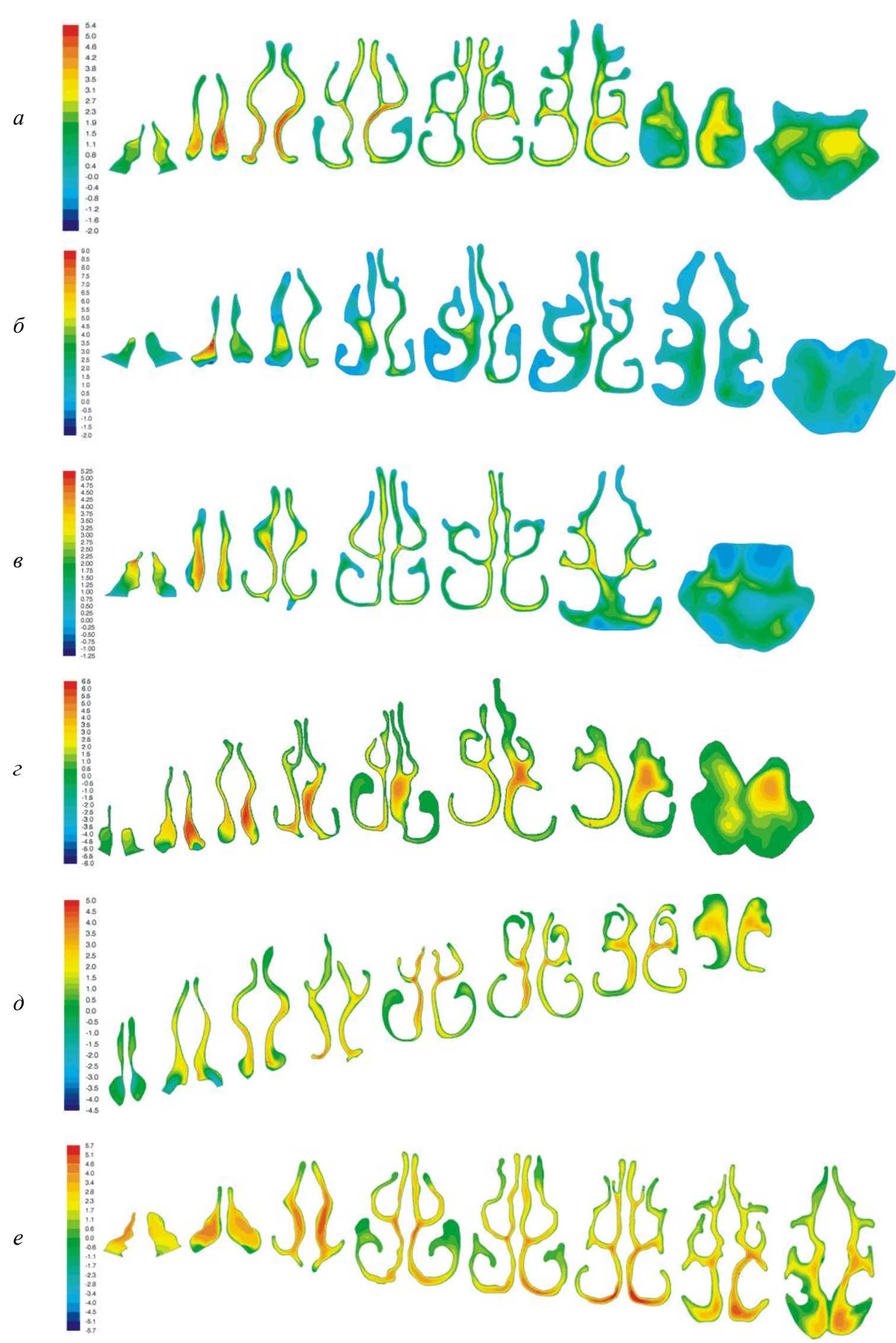

Рис. 2. Визуализация поля продольной компоненты скорости для шести моделей носовой полости 
Для последующего сравнения геометрии носовых полостей и течения в них понадобятся ринологические термины, для этого воспользуемся цитатой из работы [1]. «Полость носа разделена перегородкой на две половины - правую и левую. На противоположных перегородке стенках полости носа имеются практически горизонтальные, идущие почти параллельно костные выступы, покрытые слизистой оболочкой - носовые раковины (верхняя, средняя и нижняя, причем верхняя раковина имеется не всегда). Пространства, ограниченные боковой стенкой носа и этими раковинами, называются носовыми ходами (соответственно верхний, средний и нижний носовые ходы). Пространство между перегородкой носа и боковой поверхностью носовых раковин называется общим носовым ходом». Добавим здесь, что область на входе в носовую полость называется преддверием (вестибюлем) носа, наиболее узкое место в начале носовой полости называется носовым клапаном, а место объединения двух половин носовой полости в одну (при переходе в носоглотку) хоаной. Отметим также, что на рис. 2 первые сечения соответствуют ноздрям, их контуры не замкнуты.

Верхние носовые ходы встретились в половине случаев из рассмотренных нами тридцати, в том числе в случаях, показанных на рис. 2, $a, e$. В половине рассмотренных случаев перегородка была заметно искривлена (см. рис. 2, б, г). Правая и левая половины носовой полости могут существенно отличаться по ширине (см. рис. 2, 6,2 ), но это необязательно связано с искривлением перегородки, как в приведенных примерах. Длина носовой полости от кончика носа до хоаны, в основном, варьировалась от 7 до 8 см, минимальная длина составила 6,2 см, максимальная 8,6 см. Объем носовой полости $V$ составлял от $1,3 \cdot 10^{-5}$ до $3,1 \cdot 10^{-5} \mathrm{M}^{3}$. В двух случаях объем правой и левой половин был практически одинаков, максимальное различие достигало $37 \%$. Для демонстрируемых случаев (см. рис. $2, a-e$ ) различие составляет соответственно 16 и $34 \% ; 16$ и $24 \% ; 8$ и $13 \%$. Площадь поверхности носовой полости варьировалась от 0,012 до $0,024 \mathrm{~m}^{2}$. Средняя величина различия площадей правой и левой половин составила $3 \%$, максимальная - $11 \%$. По мере приближения к хоане высота носового хода, как правило, уменьшается. Но из рассмотренных тридцати случаев встретились два, где высота носового хода сохраняется вплоть до носоглотки. Один из них представлен на рис. 2, e.

\section{СтрУктУРА ТЕЧЕНИЯ}

На рис. 3, а показаны полученные в расчетах распределения модуля скорости (слева) и продольной компоненты скорости $V_{Z}$ (справа) на режиме вдоха при перепаде давления $\Delta p=50$ Па. Поле течения представлено в различных ракурсах с помощью ряда равноотстоящих друг от друга коронарных сечений. Эти иллюстрации дают общее представление о распределении скорости, о застойных зонах, об областях, где скорость имеет максимальное значение. Картина течения во всех носовых полостях имеет схожие черты. Поток воздуха распространяется в основном по общему носовому ходу и по прилегающим к нему областям нижнего и среднего боковых ходов. Здесь при $\Delta p=50$ Па значение продольной скорости составляет 2-4 м/с. Максимальная продольная скорость наблюдается, как правило, в области носового клапана. В самом начале вестибюля, в оконечностях боковых ходов и в узких верхушках общего хода имеются застойные зоны со скоростями, близкими к нулю, причем компонента скорости $V_{Z}$ может принимать здесь как положительное, так и отрицательное значение относительно основного потока. Значение максимальной продольной скорости при $\Delta p=50$ Па в рассмотренных вариантах составляет 5-6 м/с, на рис. $3,6-9 \mathrm{~m} / \mathrm{c}$ в чрезвычайно узком носовом клапане. Но есть носовые полости, в которых максимум скорости достигается в местах вне области носового клапана, где на пути основного потока встречается существенное сужение прохода. Например, модель на рис. 2, $\partial$ 

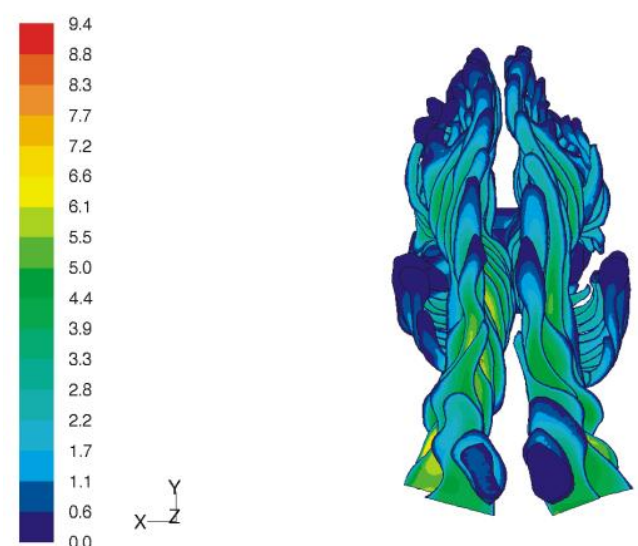

$a$

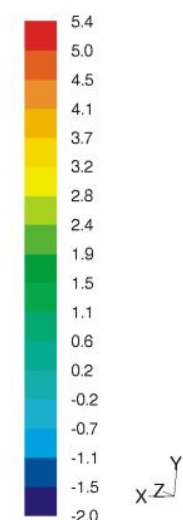

z

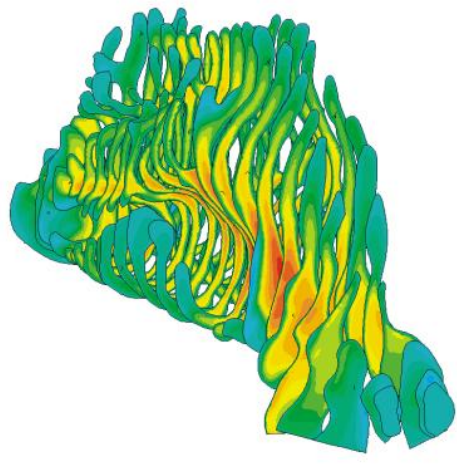

$\sigma$

Рис. 3. Визуализация абсолютного значения скорости $|V|$ и ее продольной компоненты $V_{Z}$

демонстрирует, что поток разгоняется до максимальной скорости в середине носовой полости, на рис. 2 , е почти в ее конце.

В семи вариантах отмечено обширное возвратное течение в нижнем боковом ходу. Эти варианты отличались от других вариантов тем, что общий носовой ход был очень широк. Для модели, представленной на рис. 2, б, при $\Delta p=50$ Па на рис. 4, $a$ в коронарных сечениях приведено распределение компоненты скорости $V_{Z}$ только в диапазоне отрицательных значений. Видно, что только в левой половине носовой полости с широким общим ходом (см. рис. 2, б) воздух при вдохе в нижнем боковом ходу движется в обратном направлении со скоростью 0,2-0,3 м/с. На рис. 4, б распределение $V_{Z}$ в отрицательном диапазоне приведено для одного из вариантов, не фигурирующего на рис. 2, и для удобства восприятия только для одной (левой) половины носовой полости. Анализ расчетов показал, что такое распределение скорости тесно связано с распределением температуры. Чем шире основной ход, тем меньше прогревается ядро потока от стенок носовой полости и тем вероятнее, что вдыхаемый холодный воздух может поступить в носоглотку. Но природой, видимо, предусмотрена защита в виде возвратного течения по нижнему боковому ходу, где воздух прогревается, внося свой вклад в общий нагрев носовой полости. Более детально температурное поле в носовой полости будет рассмотрено в соответствующем разделе.

Такую же защитную функцию по прогреву вдыхаемого воздуха выполняет заметное возвратное течение в широком вестибюле носа и сразу за ним, которое наблюдалось в шести вариантах. Примеры представлены на рис. 5. На рис. 4 и 5 значение $V_{Z}$ в отрицательном диапазоне по модулю меньше указанного на шкале. Дело
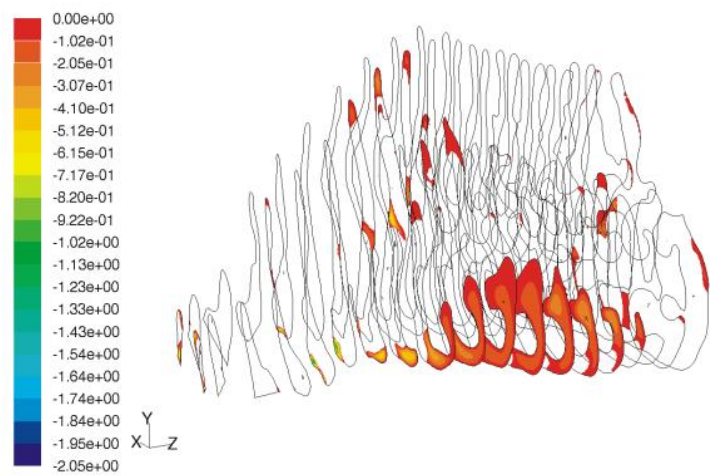

$a$

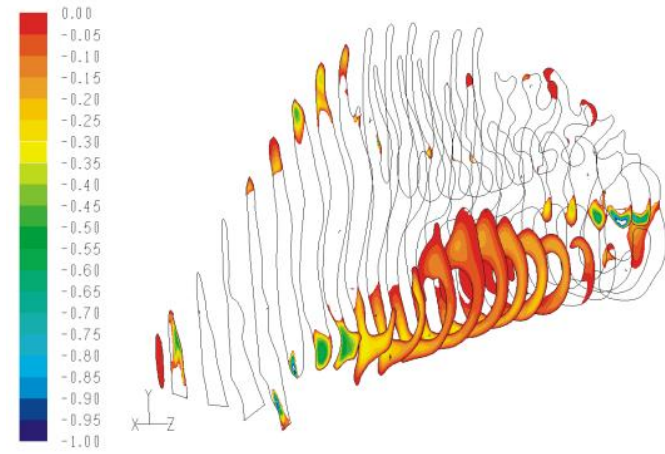

$\sigma$

Рис. 4. Возвратные течения в нижних боковых ходах 


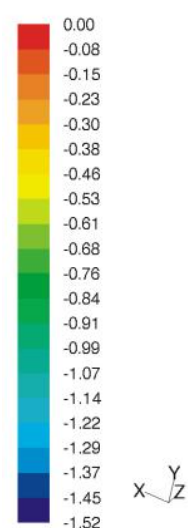

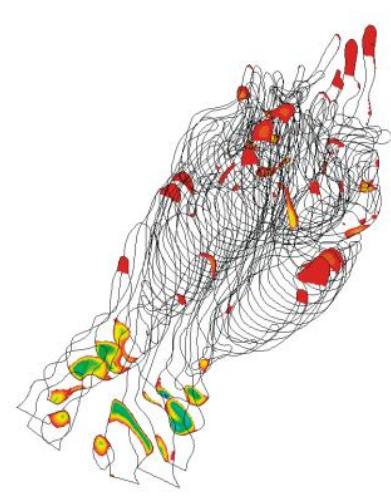

$a$

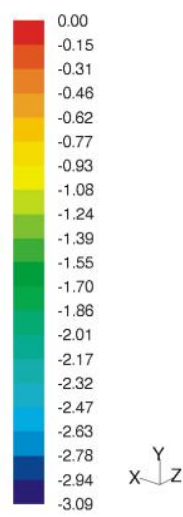

$y_{z}$

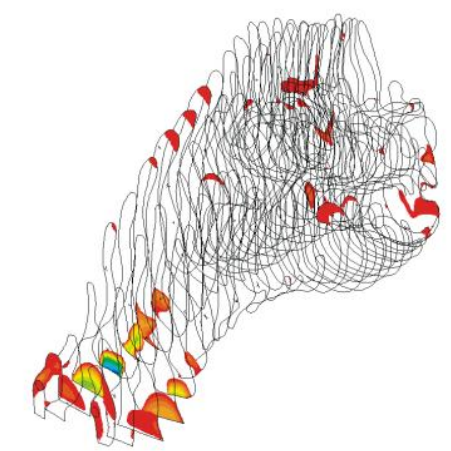

Рис. 5. Возвратные течения в вестибюле носа

в том, что области с минимальным значением $V_{Z}$, располагающиеся в вестибюле носа или сразу за ним, очень малы по размеру, так что, как правило, не попадают на опорные коронарные сечения. Для демонстрации этих областей воспользуемся изображениями в сагиттальных сечениях. Для модели, представленной на рис. 2, б, минимальное значение скорости зафиксировано внутри кончика носа, его фрагмент виден на рис. $6, a$ слева, где изображено распределение $V_{Z}$ в одном из сагиттальных сечений носовой полости.
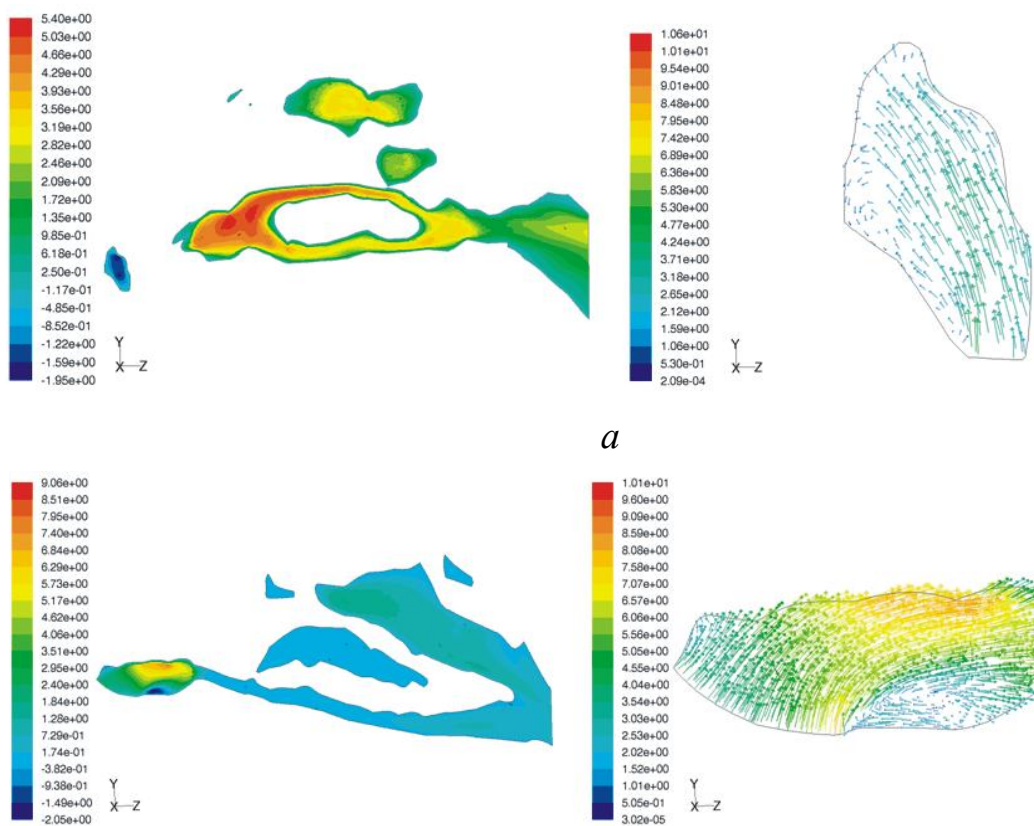

$a$
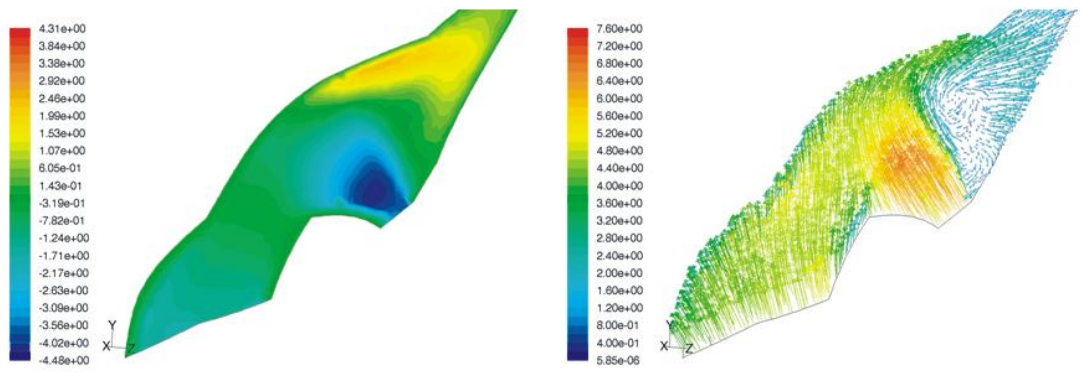

Рис. 6. Продольная компонента скорости $V_{Z}$ (слева) и векторное поле скорости (справа) - фрагменты сагиттальных сечений 
Справа на рис. 6, a этот фрагмент дан в увеличенном виде с распределением вектора скорости. Видно, что при вдохе образуется вихрь внутри кончика носа, в котором воздух нагревается и, по-видимому, частично очищается. На рис. 6 , 6 приведены аналогичные картины течения воздуха для модели, представленной на рис. 2, б. Здесь минимум скорости достигается в конце вестибюля носа, здесь также образуется вихрь, способствующий прогреву и очищению от примесей. На рис. 6, в показаны поле течения и векторное распределение скорости в районе входа в правую половину носовой полости модели, представленной на рис. 2, $\partial$.

Образуемый за ноздрей вихрь оттесняет вдыхаемый воздух от кратчайшего пути попадания в носовую полость, играя, по-видимому, также предохраняющую роль. Приведенные примеры наглядно демонстрируют защитную функцию геометрического строения входного участка носа.

\section{УДЕЛЬНЫЙ РАСХОД ВОЗДУХА}

Транспортную функцию носовой полости, т.е. способность обеспечивать легкие необходимым количеством воздуха, характеризует зависимость удельного расхода воздуха от создаваемого респираторного усилия. На рис. 7 для тридцати вариантов приведены зависимости объёмного расхода от перепада давления в носовой полости. Считается, что в среднем спокойному вдоху соответствует расход 500 мл/с. Как видно из рис. 7, для разных людей респираторное усилие, необходимое для этого значения, может составлять от 15 до 70 Па. Для большинства вариантов значение $Q=500$ мл/с достигается при $\Delta p=25-50$ Па. На рис. 7 несколько кривых лежат выше «пакета» из большинства зависимостей. Четыре из этих кривых соответствуют вариантам с большим объемом носовой полости, обладающей широкими основными ходами; две зависимости относятся к вариантам с отрицательным наклоном носовой полости (см. рис. 2, e); самая верхняя кривая соответствует экстремальному варианту так называемого «пустого носа», о котором дальше будет рассказано подробно. Ниже «пакета» лежат три кривые для самых малых по объему носовых полостей, из них самая нижняя кривая относится к варианту с большим положительным наклоном носовой полости (см. рис. 2, д). На рис. 8 значения перепада давления при вдохе,

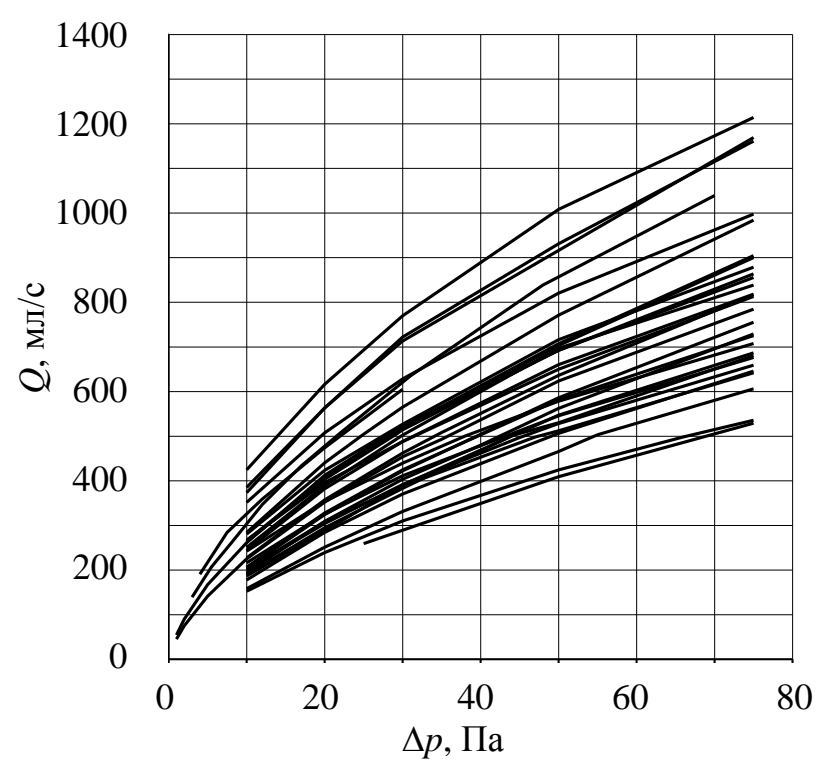

Рис. 7. Зависимость объёмного расхода воздуха от перепада давления для 30 моделей

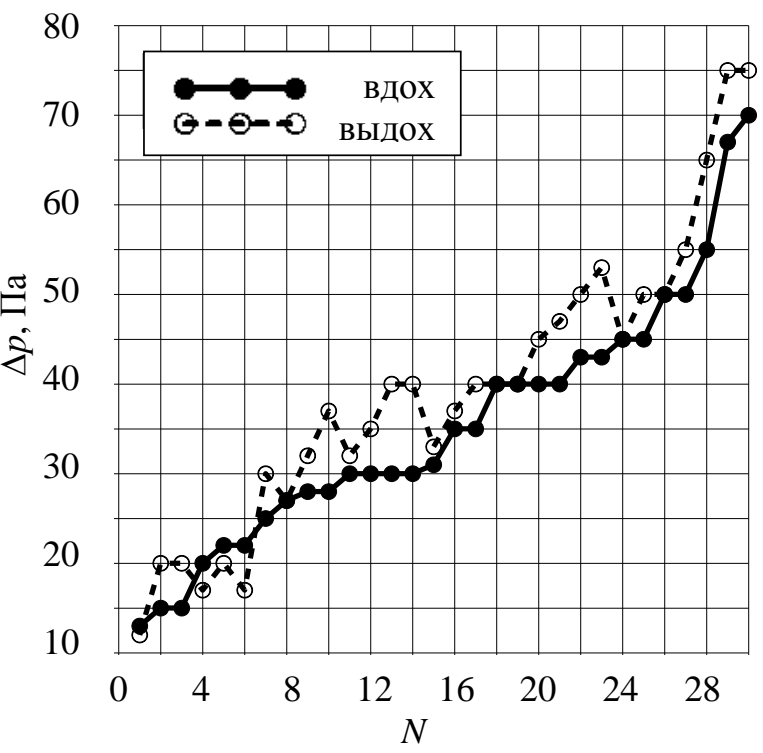

Рис. 8. Величина перепада давления при расходе 500 мл/с для 30 моделей 
соответствующие $Q=500$ мл/с, представлены на одном графике, где носовые полости пронумерованы в порядке возрастания необходимого значения $\Delta p$. Здесь же приведены соответствующие значения $\Delta p$ для режима выдоха.

На рис. 9 приведены зависимости «расход - перепад давления» для режимов вдоха и выдоха для разных вариантов. Зависимости показаны отдельно для левой и правой половин носовой полости, а также для суммарного расхода. Наиболее часто наблюдается вариант I, для которого при одинаковом респираторном усилии выдыхается меньше воздуха, чем вдыхается; или, другими словами, на одинаковый расход при выдохе требуется большее усилие, чем при вдохе (см. рис. 8). При этом зависимости для правой и левой половин носовой полости различаются. Встречаются носовые полости, для которых вдох и выдох одинаковы по усилию (II и III); реже, когда выдох «легче» вдоха (IV).
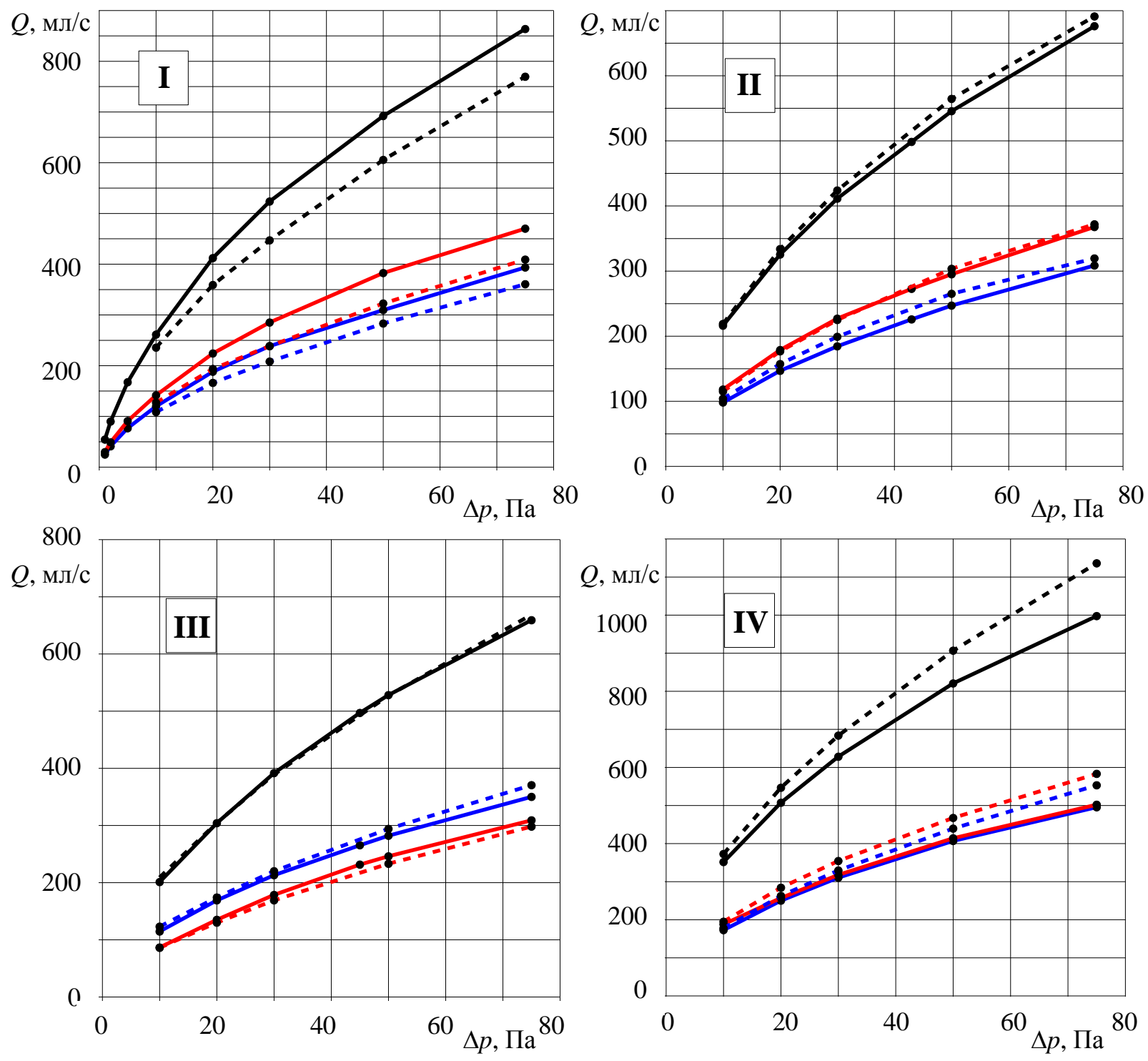

Рис. 9. Примеры зависимостей «расход - перепад давления» (синий цвет - левая половина носовой полости, красный - правая, черный - вся носовая полость) 


\section{РАСПРЕДЕЛЕНИЕ ТЕМПЕРАТУРЫ В НОСОВОЙ ПОЛОСТИ}

В расчетах на поверхности носовой полости ставилось условие постоянства температуры $T_{w}=34{ }^{\circ} \mathrm{C}$ (так же, как в работе [7], авторы которой имеют опыт прямых измерений температуры в носовой полости). На входе задавалось значение температуры наружного воздуха. Общее представление о распределении температуры при вдохе даёт рис. 10, на котором показаны поля температуры для двух носовых полостей при температуре на входе $0{ }^{\circ} \mathrm{C}$. Воздух, поступающий через ноздри в носовую полость, постепенно прогревается. Чем уже носовой ход, тем интенсивнее прогревается воздух. На рис. 10, a приведено температурное поле для носовой полости, представленной на рис. 2, б. Видно, что в этом варианте с широким общим носовым ходом в левой половине носовой полости прохладный воздух доходит до носоглотки. Температура здесь была бы еще ниже, если бы не прогрев в нижнем боковом ходе с его обширным возвратным течением (см. рис. 4, a). Рассматриваемые варианты значатся на рис. 8 под номерами $N=24$ (см. рис. 2, б) и $N=25$. На рис. 11 для них представлены распределения осредненного по коронарному сечению $T_{\mathrm{cp}}$ и минимального $T_{\min }$ значений температуры при температуре на входе $T_{\mathrm{Bx}}=23{ }^{\circ} \mathrm{C}$ (рис. $11, a)$ и $T_{\mathrm{Bx}}=0{ }^{\circ} \mathrm{C}$ (рис. $\left.11, \sigma\right)$. Распределения температуры на рис. $11, a$ приведены для наружной температуры $23{ }^{\circ} \mathrm{C}$ - некоторому среднему значению температуры, при которой проводятся томографические съемки. Самая первая точка на графиках относится к коронарному сечению внутри кончика носа. Три следующие относятся к сечениям, открытым через ноздри наружу. Минимальные значения температуры в этих сечениях равны температуре наружного воздуха.

Для двух рассматриваемых моделей, хотя длины носовых полостей несколько отличаются, значения объемов и площадей внутренней поверхности близки, расход $Q=500$ мл/с достигается при одинаковом значении $\Delta p=45$ Па. Но несмотря на близость интегральных параметров, температурные характеристики заметно различаются вследствие индивидуальных анатомических особенностей. Видно, что при $T_{\text {вх }}=23^{\circ} \mathrm{C}$ носовая полость при $N=24$ прогревается несколько меньше, чем при $N=25$ : в хоане различие равно $1{ }^{\circ} \mathrm{C}$ для средней температуры и $3{ }^{\circ} \mathrm{C}$ для минимальной. Но для $T_{\mathrm{Bx}}=0{ }^{\circ} \mathrm{C}$ это различие уже гораздо существеннее: в хоане значение $T_{\mathrm{cp}}$ составляет $28^{\circ} \mathrm{C}$ для $N=24$ против $32{ }^{\circ} \mathrm{C}$ для $N=25$, а минимальная температура для $N=24 T_{\min }=16^{\circ} \mathrm{C}$ против $\mathrm{T}_{\min }=26^{\circ} \mathrm{C}$ для $N=25$.

Заметим, что проводились расчеты с отрицательными значениями температуры наружного воздуха. Здесь они не приводятся, так как возникают сомнения по поводу неизменности геометрии носовых ходов при столь низких температурах.

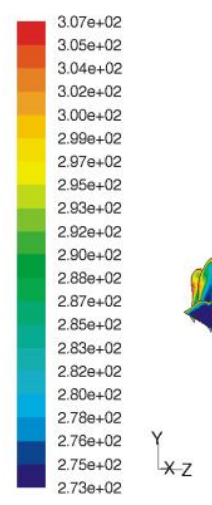

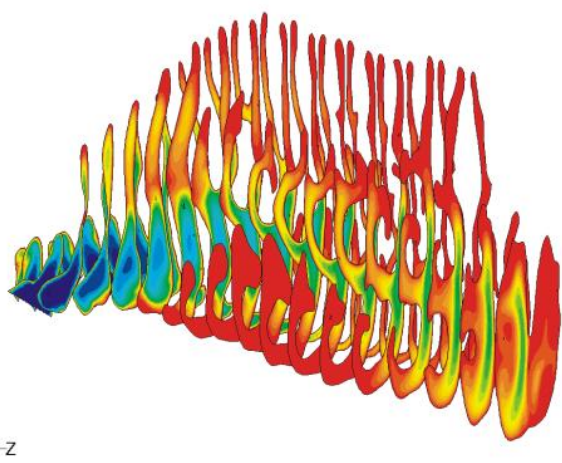

$a$

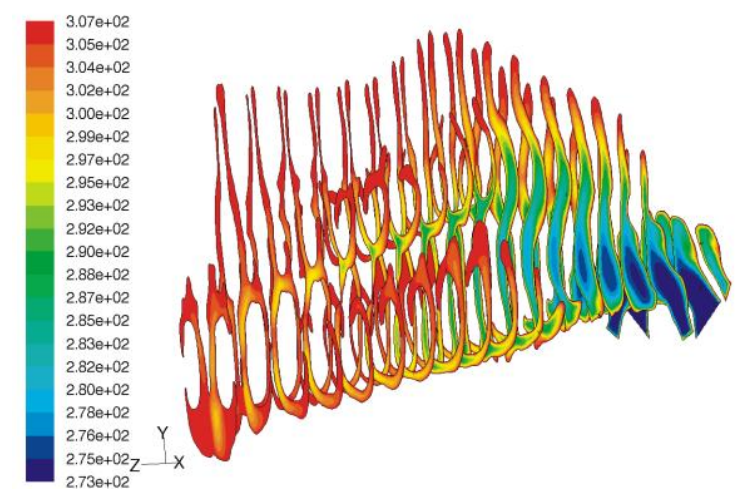

$\sigma$

Рис. 10. Визуализация поля температуры 


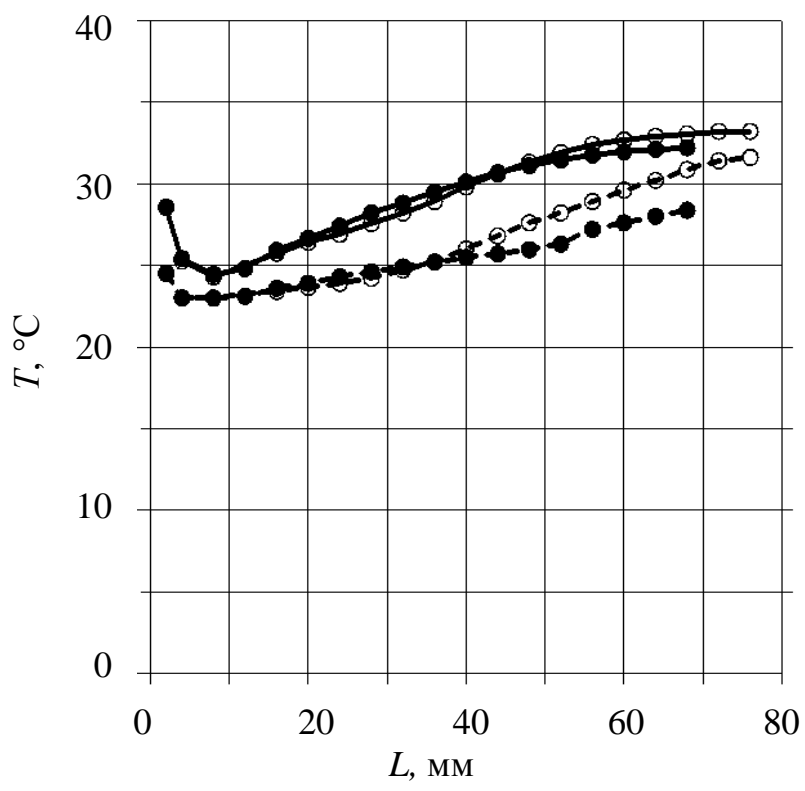

$a$

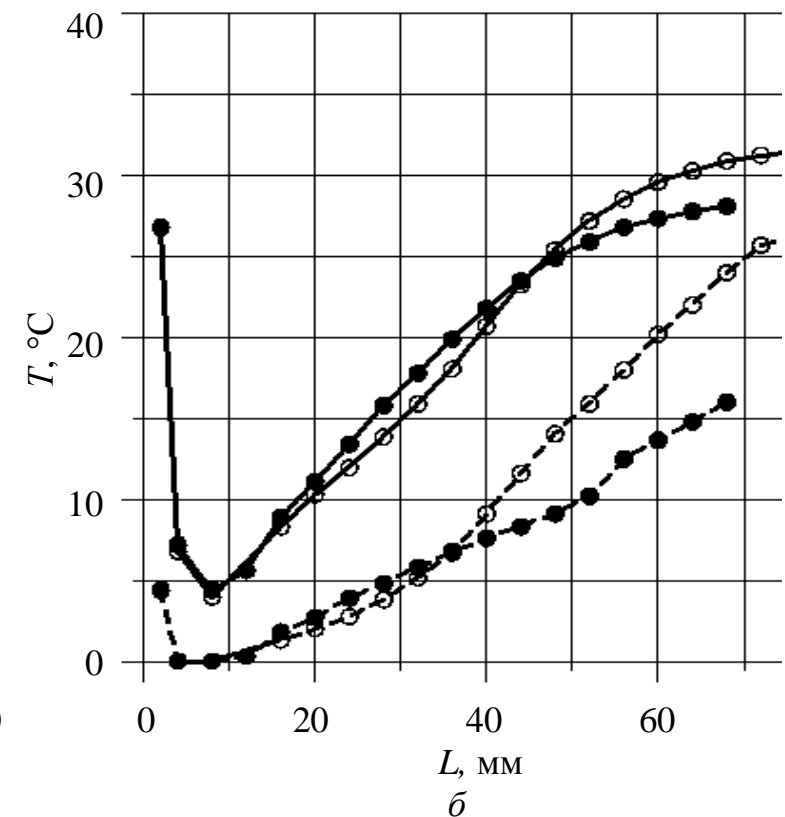

$\sigma$

Рис. 11. Распределение температуры, средней и минимальной в сечении, вдоль носовой полости при температуре наружного воздуха $T_{\mathrm{Bx}}=23{ }^{\circ} \mathrm{C}(a)$ и $0{ }^{\circ} \mathrm{C}(\sigma)$. Принятые обозначения: черные точки $-N=24$; белые точки $-N=25$; сплошные линии $-T_{\mathrm{cp}}$; пунктирные линии $-T_{\min }$

В этих расчетах получены еще более контрастные результаты. Нельзя ручаться за их полную адекватность, но можно говорить о тенденции.

На рис. 12 для всех тридцати вариантов представлены значения средней температуры в хоане при $T_{\mathrm{Bx}}=0{ }^{\circ} \mathrm{C}$ и $Q=500$ мл/с в той же последовательности, что и на рис. 8. Видно, что для большинства вариантов $30^{\circ} \mathrm{C} \leq T_{\mathrm{cp}} \leq 33{ }^{\circ} \mathrm{C}$. В большинстве своем, данные варианты представляют достаточно симметричные носовые полости с хорошо развитыми боковыми ходами, нередко основной ход в одной половине носовой

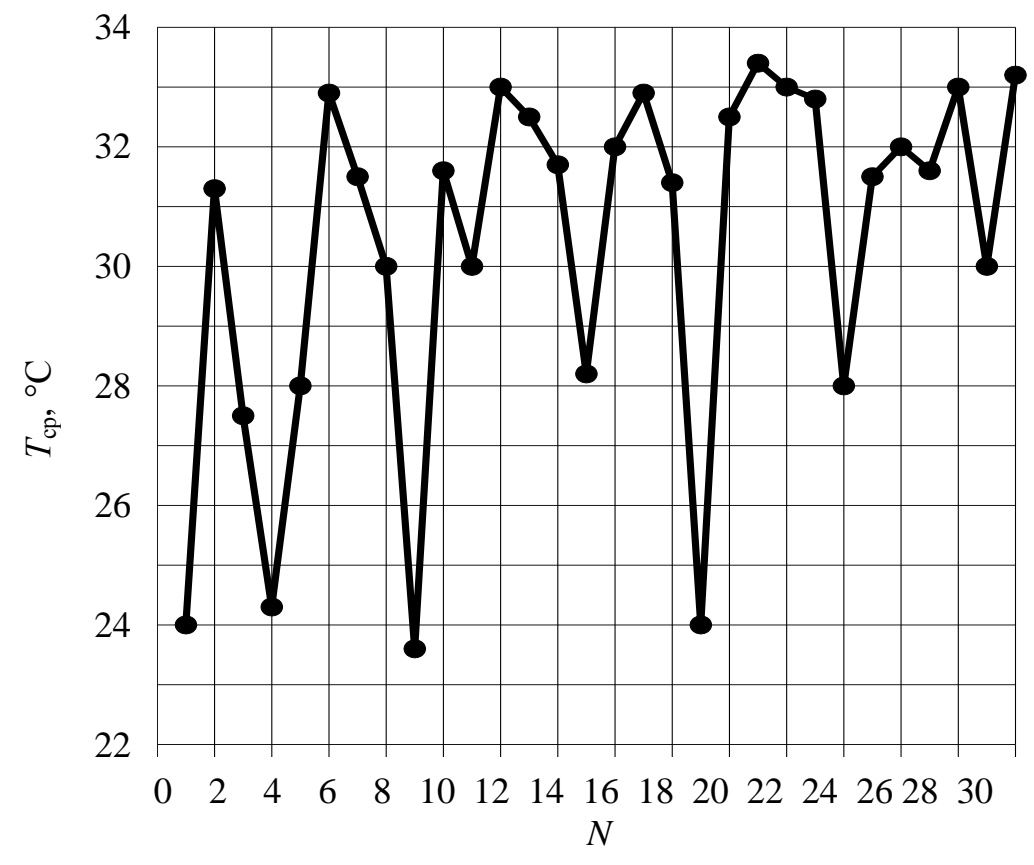

Рис. 12. Значения средней температуры в хоане при $T_{\mathrm{Bx}}=0{ }^{\circ} \mathrm{C}$ и $Q=500$ мл/с для 30 моделей 
полости может быть немного шире, чем в другой. Есть в этой большой группе отдельные случаи, которые медиками рассматриваются как патологические, например, искривление перегородки или узкие общие ходы. Но эти случаи, с точки зрения наших модельных расчетов, представляются нам вполне благополучными. На рис. 12 выделяются две группы вариантов: в четырех вариантах $\left(N=3 ; 5 ; 15\right.$ и 24) $T_{\text {cp }} \approx 28{ }^{\circ} \mathrm{C}$ и ещё в четырёх $(N=1 ; 4 ; 9$ и 19$) T_{\mathrm{cp}} \approx 24{ }^{\circ} \mathrm{C}$. Это неслучайные выбросы. Первая серия содержит варианты носовых полостей, отнесенных медиками к патологии. Эти варианты отличаются сугубо несимметричным строением носовой полости: одна половина имеет широкий основной ход, а вторая - очень узкая по причине спайки или наличия шипа на носовой перегородке. Широкая половина обеспечивает нормальное респираторное усилие, но в то же время по ней холодный воздух доходит до хоаны. Вторая серия состоит из вариантов носовых полостей после операции. Все четыре послеоперационные варианта, имеющиеся в нашем распоряжении, оказались в этой группе с самыми низкими значениями температуры на выходе из носовой полости. Разберем их отдельно подробнее.

\section{НОСОВЫЕ ПОЛОСТИ ПОСЛЕ ОПЕРАЦИИ}

На рис. 13 показаны контуры характерных сечений в коронарной плоскости для прооперированных носовых полостей. Случаи 1 и 2 демонстрируют последствия операции, в результате которой образовался так называемый «пустой нос». В случае 1 была проведена резекция носовых раковин, в случае 2 из левой половины была удалена опухоль. В случае 3 был удален костный шип на носовой перегородке. При этом данные томографии имелись как до, так и после операции. В случае 4 томография была проведена дважды - через полгода после операции и два года спустя. Этим послеоперационным случаям соответствуют номера $N=9 ; 1 ; 4$ и 19 на рис. 8 и 12. Видно, что все эти случаи выделяются очень широким общим носовым ходом. Респираторное усилие на вдохе при $Q=500$ мл/с составляет для них 28; 13; 20 и 40 Па соответственно. Зависимости «расход - перепад давления» лежат выше среднего значения на рис. 7, которое будем считать соответствующим физиологической норме по величине необходимого респираторного усилия. То есть в отношении облегчения дыхания (уменьшения респираторного усилия) хирургическое вмешательство было успешным. Но наряду

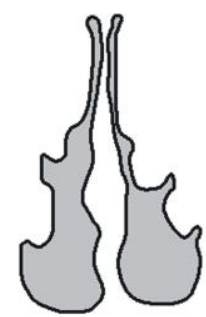

Случай 1

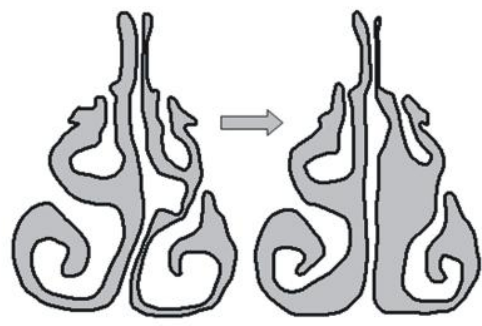

Случай 3

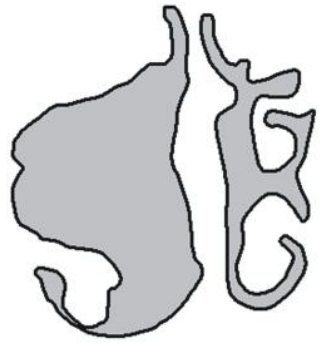

Случай 2

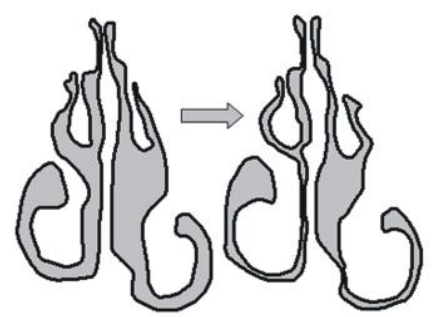

Случай 4

Рис. 13. Контуры сечений носовой полости для рассмотренных послеоперационных случаев 
с этим вдыхаемый воздух попадает в носоглотку, не успевая достаточно прогреться в носовой полости (см. рис. 12). В случае 1 резекция раковин привела к резкому расширению основного носового хода и вдобавок к исчезновению боковых ходов, которые вследствие их сравнительной узости и малых скоростей дают весомый вклад в общий прогрев. Пример случая 3 , для которого имеется дооперационный вариант томографии $(N=8)$, показывает, что произошло существенное ослабление терморегулирующей функции: средняя температура в носоглотке при $T_{\mathrm{Bx}}=0^{\circ} \mathrm{C}$ снизилась с 30 до $24^{\circ} \mathrm{C}$. В случае 4 за два года после операции носовая полость трансформировалась - общий носовой ход сузился, расход воздуха уменьшился, график зависимости «расход - перепад давления» опустился ниже, чем при физиологической норме: расход $Q=500$ мл/с достигается при $\Delta p=67$ Па $(N=29)$. Зато средняя температура в носоглотке поднялась до $30^{\circ} \mathrm{C}$. Пациент был сравнительно молод (23 г.) и, возможно, организм таким образом отреагировал на негативные последствия операции. Соответствующие показатели экстремального «пустого носа» в случае 2 появились в результате выполнения жизненно необходимой операции. Но если нет острой необходимости, нам кажется, что планировать хирургическое вмешательство следует осторожно.

\section{ЗАКЛЮЧЕНИЕ}

Два основных вывода и вытекающие из них рекомендации для практики можно сделать на основе анализа результатов численного моделирования тридцати носовых полостей реальных людей. Во-первых, форма носовых полостей и структура течения в них воздуха отличаются исключительным разнообразием. Этот факт следует учитыватьпри разработке способов доставки лекарственных веществ через нос, так как результаты этой доставки могут значительно отличаться для разных людей. Во-вторых, форма носовых ходов и распределение температуры в них тесно взаимосвязаны. Расширение носовых ходов хирургическим способом может привести к ослаблению терморегулирующей функции носа. Чтобы избежать этого, желательно реальную операцию предварять серией виртуальных операций на основе численного моделирования.

\section{БЛАГОДАРНОСТИ}

Авторы выражают признательность академику В.М. Фомину за постоянное внимание к работе.

\section{СПИСОК ЛИТЕРАТУРЫ}

1. Фомин В.М., Ветлуцкий В.Н., Ганимедов В.Л., Мучная М.И., Шепеленко В.Н., Мельников М.Н., Савина А.А. Исследование течения воздуха в носовой полости человека // Прикладная механика и техническая физика. - 2010. - Т. 51, № 2. - С. 107-115.

2. Фомин В.М., Ганимедов В.Л., Мучная М.И., Мельников М.Н., Садовский А.С., Шепеленко В.Н. Численное моделирование течения воздуха в полости носа человека с имитацией применения клинического метода передней активной риноманометрии // Прикладная механика и техническая физика. - 2012. - Т. 53, № 1. - С. 58-66.

3. Garcia G.J., Bailie N.A., Martins D.A., Kimbell J.S. Atrophic rhinitis: A CFD study of air conditioning in the nasal cavity // Journal of Applied Physiology. - 2007. - Vol. 103, № 3. - P. 1082-1092.

4. Hörschler I., Schröder W., Meinke M. Numerical analysis of the impact of the nose geometry on the flow structure. Part II: Nasal valve and lower turbinate // Computational Fluid Dynamics Journal. - 2008. Vol. 16, № 3 (26). - P. 243-260.

5. Intaek H., Scherer P.W., Mozell M.M. Velocity profiles measured for airflow throw a large-scale model of the human nasal cavity // Journal of Applied Physiology. - 1993. - Vol. 75, № 5. P. 2273-2287. 
6. Keyhani K., Scherer P.W., Mozell M.M. Numerical simulation of airflow in the human nasal cavity // J. Biomech. Engng. - 1995. - Vol. 117. - P. 429-441.

7. Lindemann J., Keck T., Wiesmiller K., Sander B., Brambs H.J., Rettinger G., Pless D. A numerical simulation of intranasal air temperature during inspiration // The Laringoscope. - 2004. - Vol. 114, № 6. P. 1037-1041.

8. Rhee J.S., Cannon D.E., Frank D.O., Kimbell J.S. Role of virtual surgery in preoperative planning. assessing the individual components of functional nasal airway surgery // Arch. Facial. Plast. Surg. - 2012. - № 14 (5). P. 354-359.

9. Segal R.A., Kepler G.M., Kimbell J.S. Effect of differences in nasal anatomy on airflow distribution: a comparison of four individuals at rest // Annals of Biomedical Engineering. - 2008. - Vol. 36, № 11. P. 1870-1882.

10. Shen Yu, Xiu-Zhen Sun, and Ying-Xi Liu. Numerical analysis of the relationship between nasal structure and its function // The Scientific World Journal. - 2014. - Vol. 2014, Article ID 581975. - P. 1-6.

11. Wen J., Inthavong K., Tian Z.F., Tu J.J., Xue C.L., Li C.G. Airflow pattern in both sides of a realistic human nasal cavity for laminar and turbulent conditions // Proceedings of 16th Australasian Fluid Mechanics Conference, 2-7 December 2007. - Gold Coast, 2009. - P. 68-74.

12. Wilcox D.C. Formulation of the $k$-omega turbulence model revisited // AIAA Journal. - 2008. - Vol. 46, № 11. - P. 2823-2838.

13. Zhang Z., Kleinstreuer C. Low Reynolds number turbulent flows in locally constricted conduits: a comparison study // AIAA Journal. - 2003. - Vol. 41, № 5. - P. 831-840.

\section{AIR FLOW IN THE HUMAN NASAL CAVITY. THE RESULTS OF MATHEMATICAL MODELLING}

\section{V.L. Ganimedov, M.I. Muchnaya, A.S. Sadovskii (Novosibirsk, Russia)}

The complex structure of the human nasal cavity makes it practically impossible to study the air flow by experimental imaging and flow diagnostics; so numerical modelling is widely used for this purpose now. These studies are relevant in view of the development of methods for inhaled drug delivery; moreover, they can be used to carry out virtual surgery. A technique for studying the air flow in the human nasal cavity through mathematical modelling by using the ANSYS package is developed at the Khristianovich Institute of Theoretical and Applied Mechanics of the Siberian Branch of the Russian Academy of Sciences. The three-dimensional geometry of the nasal cavity is constructed on the basis of clinical tomographic images. The GRAPHER and GAMBIT programs are used for geometry definition. In this geometric model of the nasal cavity, the flow is calculated in the gasdynamic part of the ANSYS (FLUENT) package on the basis of Reynolds-averaged NavierStokes equations with the $k-\omega$ turbulence model. By means of a time-dependent method, a quasi-stationary solution is found for a prescribed pressure difference between the entrance and exit of the nasal cavity. Thirty models of the nasal cavities of real adult people are constructed. Extreme diversity of shapes and, as a consequence, significant differences in the flow patterns should be noted. The calculation results are analyzed to search for regularities and individual features. The integral characteristics, such as the dependence of the volume flow rate on the pressure difference and the distributions of the minimum and average values of temperature along the nasal cavity depending on the temperature of inhaled air, are compared. It is shown that it is not sufficient to know only the flow rate characteristics of the nasal cavity for determining the physiological norm; it is also necessary to consider the thermal-control function.

Key words: numerical simulation, human nasal cavity, flow structure, flow field visualization, respiratory effort, temperature distribution. 